\title{
MEK Family Gene Mutation
}

National Cancer Institute

\section{Source}

National Cancer Institute. MEK Family Gene Mutation. NCI Thesaurus. Code C136422.

A change in the nucleotide sequence in a MEK family gene. 